\title{
Barriers and Facilitators of Art Adherence Among Hiv Positive Patients in Community Client-led Art Delivery Groups at Lira Regional Referral Hospital
}

Rogers Isabirye

Lira University

Steven Sean Puleh ( $\nabla$ spuleh@lirauni.ac.ug)

Lira University

Didan Jacob Opii

Lira University

Sharon Opio-Ekit

Lira University

Alice kawomera

Lira University

Luke Lokiru

Lira University

Robert Isoke

Lira University

Joachim Ssenkaali

Lira University

Edward Kumakech

Lira University

Celestino Obua

Mbarara University of Science and Technology

\section{Research Article}

Keywords: adherence, Community Client Lead ART Delivery, barriers, facilitators, HIV/AIDS

Posted Date: March 3rd, 2021

DOl: https://doi.org/10.21203/rs.3.rs-200016/v1

License: (c) (1) This work is licensed under a Creative Commons Attribution 4.0 International License.

Read Full License 


\section{Abstract}

Background: The community client-led ART delivery groups (CCLADs) was introduced as one of the strategies to better serve individual needs and reduce unnecessary burdens on the health system. However, no study has comprehensively explained what are the drivers and barriers of CCLADs in improving treatment outcomes. This study sought to assess the barriers and facilitators of ART adherence among HIV positive patients attending CCLADs at Lira Regional Referral Hospital (LRRH), Lira District.

Method: We employed a mixed methodology involving 150 study participants between July to August 2020. Quantitative data was obtained from all the participants that were picked through systematic random sampling using a semi-structured questionnaire. Data was entered into SPSS version 23.0 and analyzed at $95 \%$ level of significance. We conducted 25 in-depth interviews guided by a checklist. Qualitative data was analyzed through thematic content analysis of major themes that emerged from participants' responses.

Results: Our study found that majority $94.7 \%(142 / 150)$ of the respondents had an optimal adherence $(100 \%)$ calculated retrospectively based on a 4-day pill uptake recall. In addition, $39.3 \%(59 / 150)$ of participants in CCLADs at LRRH had missed taking a pill in a period of at least 4 weeks. Among all the 104 respondents that had ever missed a medication, the most frequent reason $(35 \%(35 / 104))$ for missing a medication was travelling far away.

From qualitative data, social support, patient self-motivation, health education and counselling and guidance were the major facilitators to adherence. On the other hand, lack of food, stigma, forgetfulness, stress, unfair hospital staffs and cultural beliefs were the major perceived barriers to ART adherence.

Conclusion/recommendation: Good adherence was attributed to availability of ART at the clinic and an efficient delivery strategy. More health care follow up interventions should be designed to ensure total pill uptake by PLWHIV in communities.

\section{Introduction}

Globally, 37.9 million people live with HIV, 1.7 million people become newly infected yearly and about 32.0 million people died from AIDS-related illnesses since the start of the epidemic (UNAIDS, 2019). At the end of 2018 , an estimated $79 \%$ of PLWHIV knew their status, about $62 \%$ were receiving ART and only $53 \%$ had reached viral load suppression far below the 95, 9595 target by 2030 (UNAIDS, 2014). The UNAIDS (2019) report shows that in Uganda, 1,400,000 people were living with HIV by the end of 2018 of which 53,000 people were newly infected while 23,000 people had died from AIDS-related illnesses. Seventy-two percent $(72 \%)$ of the People living with HIV (PLWHIV) in the same year were on treatment of whom only $64 \%$ were virally suppressed (UNAIDS, 2019). The HIV epidemic still accounts for a high mortality rate despite interventions put in place. Full ART adherence is central to good treatment outcomes, however, it still remains a challenge among HIV positive patients to date (Ridgeway et al., 2018). 
In Sub-Saharan Africa one of the regions most burdened with HIV/AIDS, ART service delivery puts a lot of pressure on the health system. The heavy burden translates into higher cost, congestions, and long waiting times due to the long waiting lines (Geng \& Holmes, 2019). The World Health Organization (WHO) and major donors recommended differentiated HIV service delivery as a novel evidenced based strategy to reduce congestion at the facility ART clinic, promote meaningful involvement of PLWHIV, and to enhance patients' self-management (Grimsrud et al., 2016). The differentiated delivery models included Community Client-Led ART Delivery Groups (CCLADs), Community Drug Distribution Points (CDDPs), Facility-Based Groups (FBGs), and Facility-Based Individual Management (FBIM). In Uganda CCLADs has been implemented as one of the differentiated service delivery (DSD) model since 2017 by the ministry of health (Roy et al., 2019),(Zakumumpa et al., 2020). CCLADs as a client centered approach is meant to improve on the quality of care through provision of ART delivery while acknowledging specific drivers and barriers in a way that empowers them to manage their disease with the support of the health system (UNAIDS, 2015).

Each CCLAD group consists of between 4 to 6 HIV positive patients who come from the same community. An expert client is identified by group members and made the group leader. The collection of ART regiments at each refill for all the group members from the facility is done by only one member who then distributes to the others. However, no study has explicitly described the drivers and barriers of CCLADs in improving adherence and treatment outcomes. In addition, HIV differentiated care might be influenced by many factors such as complexity around patient's preferences among others (Grimsrud et al., 2016, Barker et al., 2017 and Roy et al., 2019). It is against this background that this study assessed the barriers and facilitators of ART adherence among HIV positive patients at Lira infectious disease clinic (LIDC) in LRRH, Lira District.

\section{Methods}

\section{Study design/setting}

We conducted a cross sectional study that employed both quantitative and qualitative methods (mixed methods) for data collection between July-August 2020.

We conducted the study at Lira Regional Referral Hospital located in Lira city, northern Uganda. Lira is approximately 340 kilometers from Kampala, the capital city of Uganda. Lira Regional Referral Hospital has a catchment area that includes Amolatar, Apac, Dokolo, Lira, Kole, and Oyam Districts.

\section{Study population}

We studied HIV positive patients receiving ART in the CCLADs models at LRRH. All participants were adults ( $\geq 18$ years) who voluntarily agreed to receive ART in CCLADs, and they included both males and females.

\section{Sample size estimation}


The sample size of the study was estimated following the methods for estimating sample size of cross sectional studies and we used Yamane's (1967) formula of sample size determination to determine our study sample size. $n=N /\left(1+\mathrm{Ne}^{2}\right)$

Where; $\mathbf{n}$ is the sample size, $\mathbf{N}$ is the size of the population (219), $\mathbf{e}$ is the margin of error (5\%). We estimated our study population at 150 participants including an allowance for non-response of $10 \%$.

\section{Sampling criteria}

For quantitative data: We first calculated the sampling interval by dividing the total number of clients in CCLADs attending LIDC (219) by our sample size (150). In this case, the sampling interval of 2 was determined. We then selected a number between 1 and the sampling interval 2 from the random number table. Client number 2 was therefore the first client interviewed. We then counted down the list starting with client number 2 and selected each $2^{\text {nd }}$ client.

All the selected clients were contacted for recruitment in the study through linkage facilitators.

For qualitative data: We purposively sampled clients in CCLADs to obtain qualitative data through in depth interviews.

\section{Data collection tool(s)}

The study tool for quantitative data was modified from the Adult AIDS Clinical Trial Group (AACTG) adherence baseline questionnaire as used in a study by Hansana et al., 2013. In addition, we used an Indepth interview guide modified from the interview guide by Kvale (1996) used in a study to assess SocialEcological View of Barriers and Facilitators for HIV Treatment Adherence.

The study tools were pretested on a group of 15 HIV patients at Amach health center IV and the information obtained was used to modify the study tools.

The AACTG tool collected information on; Socio-demographics (age, sex, marital status, occupation, location of residence) and Pill uptake information (last time of missing medication, reasons for missing medication, number of pills taken per day, time of taking medicine, motivation to take medicine.

\section{Data collection method/criteria}

Quantitative data: The researchers administered the semi-structured questionnaires individually per respondent. The interviews took on average 10 minutes each.

Qualitative data: Interviews were conducted from the participants and the audios were recorded during the interviews. The interviews were conducted in the language best understood by respondent (English or Luo). Information from the participants were obtained by use of an interview checklist. The interviews took an average of 20 minutes each. 


\section{Data management and analysis.}

Quantitative data: Administered questionnaires were checked for completeness by researchers immediately after filling in responses from the participants. The data collection team further met at the end of every data collection day to check for any missing data from the filled questionnaires.

The data entry screen was created in SPSS version 23.0 and cleaned. Data entered was analyzed using SPSS version 23.0. Continuous variables like age; were summarized using mean, median, range and standard deviation. The relationships between the dependent and independent variables were established on odds ratios with a $95 \%$ confidence interval.

Qualitative data: Qualitative data was stored in audio recorders during interviews and was transcribed, read multiple times for data familiarization (Miles et at., 1994). The data was coded manually and codes were merged following consensus from the authors of the study then summarized into themes. Thematic analysis was done based on major themes that emerged from the responses obtained during the interviews.

\section{Results}

The section gives details of the findings on socio-demographic characteristics, patients' pill uptake adherence, and the perceived barriers and facilitators to adherence, obtained after data analysis.

In our study, 150 participants were identified and interviewed for obtaining information on adherence giving a response rate of $100 \%(150 / 150)$. The participants were aged ranging from 22 to 70 years with a mean age of $44.9(S D=11.25)$. Majority $76 \%(114 / 150)$ of the participants were females with $62.0 \%$ (93/150) being peasant farmers (Table 1$)$.

Table 1: Socio-demographic characteristics of HIV positive clients in CCLADs at LRRH (July, 2020) 


\begin{tabular}{|cll|}
\hline Characteristic & Frequency n (\%) & P-values \\
\hline Sex & & 0.434 \\
\hline Male & $36(24.0)$ & \\
\hline Location of residence & 0.379 \\
\hline Urban & $88(58.7)$ & \\
\hline Rural & $62(41.3)$ & $0.027^{\star}$ \\
\hline Occupation & & \\
\hline Farmer & $93(62.0)$ & \\
\hline Business & $48(32.0)$ & \\
\hline Civil servant & $9(6.0)$ & \\
\hline Marital status & & \\
\hline Single & $8(5.3)$ & \\
\hline Married & $83(55.3)$ & \\
\hline Divorced & $15(10.0)$ & \\
\hline Widowed & $44(29.3)$ \\
\hline Level of education & \\
\hline No education & $25(16.7)$ \\
\hline Primary & $92(61.3)$ & \\
\hline Secondary & $25(16.7)$ & \\
\hline Tertiary & $8(5.3)$ & \\
\hline & & \\
\hline
\end{tabular}

*significantly associated to adherence

\section{Adherence}

We assessed adherence by looking at the participants' pill uptake information based on a 4 day pill uptake recall. Adherence was considered as optimal (at least $95 \%$ ) and sub-optimal $<95 \%$. Majority $94.7 \%(142 / 150)$ of the participants had an optimal adherence of $100 \%$ (Figure 1). From the qualitative data, we identified four themes as facilitators of adherence. These were social support, patient selfmotivation, health education and counselling and guidance. Most of the participants said they would offer social support such as encouragement and advice to their friends in the group who want to stop or has already stopped taking their ARVs. One of the respondents said that; 
"I give the person company if near me and I continue advising him/her because they should live a normal life". Female, R6, 53 years.

In this study about $30.7 \%(46 / 150)$ of the respondents had never missed taking their medication.

However, a total of $39.3 \%(59 / 150)$ of the respondents reported having missed to take their medication in a period of more than four weeks. In addition, majority $90.7 \%$ (136) of the respondents said they were self-motivated to take their medications (Table 2). From the qualitative data, most of the participants interviewed reported that the HIV medication has been very helpful to them and because of this, they feel like the medication should not be discontinued. In addition, some of the respondents reported desires to continue living and caring for their family as their motivation to take ARVs.

"I realized the goodness of this drug because it was making me stronger and healthier. I keep on taking ARVs because it is making me able to live, pay school fees, and farm". Male, R3, 53 years.

Some of the patients reported that they continued to take their ARVs because they could feel a lot of discomfort before they started taking ARVs and found relief only after starting to take ARVs as one female respondent puts it

"......... how my body used to pain me to the extent that I thought I was going to die". Female, R18, 53 years

Table 2: Pill uptake information for HIV positive patients in CCLADs at LRRH (July, 2020) 


\begin{tabular}{|c|c|c|}
\hline Characteristic & Frequency(n) & Percentage (\%) \\
\hline \multicolumn{3}{|l|}{ Last time of missing medication } \\
\hline In past 3 days & 6 & 4 \\
\hline In the past week & 11 & 7.3 \\
\hline 1-2 weeks ago & 12 & 8 \\
\hline 2-4 weeks ago & 16 & 10.7 \\
\hline More than 4 weeks ago & 59 & 39.3 \\
\hline Never miss medication & 46 & 30.7 \\
\hline \multicolumn{3}{|l|}{ Reason for missing medication } \\
\hline Travelled far away & 35 & 33.7 \\
\hline Medication got finished & 6 & 5.8 \\
\hline Busy & 22 & 21.4 \\
\hline Forgot & 16 & 15.4 \\
\hline Wanted to avoid side effects & 25 & 24 \\
\hline \multicolumn{3}{|l|}{ Number of pills taken per day } \\
\hline 1 & 145 & 96.7 \\
\hline 2 & 3 & 2 \\
\hline 3 & 1 & 0.7 \\
\hline 5 & 1 & 0.7 \\
\hline \multicolumn{3}{|l|}{ Time of taking medication } \\
\hline Morning & 63 & 42 \\
\hline Evening & 87 & 58 \\
\hline \multicolumn{3}{|l|}{ Motivation to take medication } \\
\hline Self & 136 & 90.7 \\
\hline Family member & 13 & 8.7 \\
\hline Others & 1 & 0.7 \\
\hline \multicolumn{3}{|l|}{ Adherence on 4 day recall } \\
\hline Optimal (100\%) & 142 & 94.7 \\
\hline
\end{tabular}




\begin{tabular}{|lll|} 
Sub-optimal $(<100 \%)$ & 8 & 5.3 \\
\hline
\end{tabular}

Association between socio-demographic characteristics and adherence to the four day pill uptake recall

Our data shows that occupation of the respondents was significantly associated with adherence to the four day pill uptake recall (Table 3 ).

Table 3: Association of socio-demographic characteristics with adherence on a four-day pill uptake recall, for HIV positive patients in CCLADs at LRRH. 


\begin{tabular}{|c|c|c|c|}
\hline \multirow[t]{2}{*}{ Characteristic } & \multicolumn{2}{|l|}{ Adherence count } & \multirow[t]{2}{*}{ P-value } \\
\hline & Suboptimal (75\%) & Optimal (100\%) & \\
\hline \multicolumn{4}{|l|}{ Sex } \\
\hline Male & 1 & 35 & 0.434 \\
\hline Female & 8 & 107 & \\
\hline \multicolumn{4}{|l|}{ Level of education } \\
\hline No education & 0 & 25 & 0.462 \\
\hline Primary & 5 & 87 & \\
\hline Secondary & 2 & 23 & \\
\hline Tertiary & 1 & 7 & \\
\hline \multicolumn{4}{|l|}{ Location of residence } \\
\hline Urban & 3 & 85 & 0.379 \\
\hline Rural & 5 & 57 & \\
\hline \multicolumn{4}{|l|}{ Marital status } \\
\hline Single & 0 & 8 & 0.528 \\
\hline Married & 3 & 80 & \\
\hline Divorced & 1 & 14 & \\
\hline Widowed & 4 & 40 & \\
\hline \multicolumn{4}{|l|}{ Occupation } \\
\hline Peasant farmer & 2 & 91 & $0.027^{\star}$ \\
\hline Business & 6 & 42 & \\
\hline Civil servant & 0 & 9 & \\
\hline
\end{tabular}

\section{*(statistically significant to adherence)}

\section{Barriers and facilitators to adherence}

We conducted 25 in-depth interviews exploring patient's perceived barriers and facilitators to ART adherence among CCLADs members at Lira Regional referral hospital. The findings under facilitators were categorized under themes; social support, patient motivation and health facility facilitators and 
findings of barriers were categorized under the themes; lack of food, health facility barriers, stigma, patient feelings, socio-cultural barriers.

\section{Facilitators of ART adherence.}

\section{Social support.}

Most of the participants said they would offer social support as a form of encouragement and advice to their colleagues in the group who want to stop or has already stopped taking their ARVs. Some of the respondents would give examples of themselves, how they were able to cope with HIV and its medications. One reported that

"I give the person company if near me and I continue advising him/her because they should live like you". Female, R6, 53 years.

In addition, the CCLAD model ensures that on person brings the drugs for the rest. One patient added that she would make sure the ARVs are available for their friends who don't feel like they should continue taking ARVs. She said that;

"If that person does not want to collect his or her ARVs, I can offer to collect for him or her from the hospital". Female, R13, 54 years

\section{Patients' self-motivation.}

Most of the participants interviewed reported that the HIV medication has been very helpful to them and because of this, they feel like the medication should not be discontinued. In addition, majority of the respondents reported desires to continue living and caring for their families as their motivation to take ARVs. One respondent said that;

"I realized the goodness of this drug because it was making me stronger and healthier. I keep on taking ARVs because it is making me able to live and pay school fees, farm". Male, R3, 53 years.

Furthermore some of the patients reported that ARVs provided them with a lot of relief, since the level of pain reduced from the time they were initiated into ART as one female respondent puts it;

"........ how my body used to pain me to the extent that I thought I was going to die". Female, R18, 53 years

Counselling and guidance. A relatively high number of respondents said they continued with their medication because the health workers would remind them of the importance of taking the drugs at every drug refill date. Counselling and guidance is very encouraging because sometimes we hard no hope. One patient said

"It was dr.......... who convinced me and I started again and I realized I could live again and I continued till now." Female, R2, 40 years. 
Most of the respondents reported that they continued to take their ARVs because they kept remembering the instructions and lessons given to them from the hospital. Additionally, most of those who missed their ARVs resumed taking their drugs because they remembered they were not supposed to skip taking their medication. Reported

"I continue taking ARVs because I always recall the instruction given to me from the hospital and also, I want to live a better life". Female, R21, 39 years

\section{Barriers to ART adherence.}

\section{Lack of food}

Majority of the respondents reported that having nothing to eat made them unable to take their medication. They reported that they must always eat food prior to taking their HIV medication. Nonetheless taking the medication on an empty stomach would make the drug to disturb them. A female respondent reported

“ I feel like it is twisting my intestines". Female, R23, 38 years.

\section{Unfair health workers}

Some patients in CCLADs missed their ARVs due to unfair treatment from some staffs at the clinic. Most respondents reported the health workers were very harsh in handling them. They insulted them with abusive and unkind words. This forced them to stop taking their ARVs. One participant said that

"When I started taking ARVs, I did not want because I found a nurse who shouted at me with my daughter and I left it for one year" Female, R2, 45 Years.

\section{Forgetfulness.}

Some of the respondents missed their ARVs because they forgot that they had to take their medicines. This was mostly resulting from being occupied with activities in the garden. By the time they realized, it was past their time for taking their ARVs. A participant reported

"I did not do this intentionally, I just forgot, but I cannot miss like for two or three days". Female, R23, 29 years.

\section{Stigma}

Some men who are HIV positive and not in CCLADs shy away from refilling their ARVs. They consequently begin to share ARVs with their wives in CCLADs. This was reported that it makes these women to miss taking their ARVs as the medications would get finished before their refill dates. Additionally it was reported those who had not disclosed to anybody outside the group about their HIV 
positive status could feel bad and at times, stopped taking their medication whenever they could hear anybody talk about their HIV status. This was done in attempt to prove that they were not positive.

"Sometimes people can point at us that we are taking HIV medication and other people can abuse and this can anger us". Female, R11, 52 years

\section{Stress}

A few respondents reported that they missed their medication in the past because they were so stressed and frustrated with the negative treatment they got from the community and from conflicts with friends and family. Some respondents added that if they got angered due to misunderstandings in the community or with family members, they would not see meaning in taking their drugs. One of the patients reported

"A sister to my husband has been abusing my daughter since she acquired HIV from me and this had made me very sad because my daughter wanted to commit suicide and it discouraged me from taking my medication" Female, R09, 37 years

\section{Socio-cultural barriers.}

Some few HIV positive patients in CCLADs reported use of traditional herbs to cure HIV as the most common belief and practice hindering the consistent use of ARVs. One participant said

"there was a friend of mine who said they are not supposed to take a particular drug for so long and she went to a herbalist and started taking herbs leaving ARVs and she died" Female, R24, 50 years

Another respondent said those without the knowledge of treatment options still continue using the harmful traditional methods to cure HIV. He reported

".............still stuck on cultural ways and beliefs such as using traditional herbs for curing HIV which can discourage him/her from taking their ARVs." Male, R22, 45 years

\section{Discussion}

In our study, most of the participants in CCLADs had good adherence as evaluated by a four-day pill uptake re-call. The good adherence could be attributed to many factors such as availability of ART drugs, the efficient delivery strategy, social support, health education, counselling and guidance. The CCLADs model minimizes congestion at the HIV clinics and improve accessibility to ART (Kagimu D, Egessa J, Oucul $L$, 2018). This result was in agreement with findings of a descriptive cross sectional study by Mugerwa et. al 2019, that showed a significant relationship between joining CCLADs and improvement to drug accessibility and adherence. Most respondents were peasant farmers who in Uganda are associated with poor socio-economic status. The clients reported that they would take their drugs before work since 
it could give them energy to work in their gardens. Evidence from elsewhere also showed giving of energy was a positive patient perceived benefit of ARVs (Nyogea et al., 2015 and Mills et al., 2006).

In this study social support was identified as a major facilitator of adherence. This is because social support minimizes on stigma and increases the level of confidence and accountability to one another. Social support enabled many of the respondents to continue taking their drugs eventually improving adherence to ART. This findings are in agreement with data from elsewhere which found that social support was a key facilitator of ART adherence (Bezabhe et al., 2014) and (Wasti et al., 2012).

Additionally, health education was identified as one of the major drivers of adherence. Health education plays a major role in ensuring that patients know about their condition, available medical services and options regarding treatment in order to make an informed decision. When the clients have been made knowledgeable, they make better decision regarding when and how they receive healthcare. Our findings are related to those from a similar study done in Tanzania (Nyogea et al., 2015) which found that being informed about the medication was a key factor in increasing the levels of adherence among HIV positive patients.

Furthermore, our study cited patient self-motivation as another facilitator of adherence to ART. The selfdrive is very important in enhancing a patient's attitude towards positive living and motivates them to take their drug on time without forgetting. This was comparable to the findings of the study conducted in southern Africa (Kagee et al., 2012) which also highlighted patient self-motivation as an enabler of good ART adherence.

Our findings showed that majority of patients had substantial level of adherence on a four-day recall, but a huge proportion would forget to take their medication as time increases. Qualitative findings showed forgetfulness as one of the major barriers. When patients forget to take their medication, an optimal adherence is not attained (Nawa et al., 2008 and Nyogea et al., 2015). This finding is comparable to a study done in Brazil, Brigido et al., 2001 and Mills et al., 2006) which also cited forgetfulness as a barrier to ART adherence.

Our findings also showed lack of food as another barrier to ART adherence. Lack of food is a barrier because it is hard to take drugs on an empty stomach, because this would lead to adverse drug reactions, like diarrhea, nausea and vomiting and body weaknesses. Evidence from elsewhere also showed lack of food as a barrier to Art adherence (Nawa et al., 2008, Weiser et al., 2014 and Bezabhe et al., 2014).

Furthermore, stigma that came through fear of being noticed taking a medication, mainly on social activities like traditional marriages and prayer seminars was a barrier. Stigma if not addressed causes missing of medication, poor care involvement like turning up for drug refill and viral load testing, and may consequently lead to loss of follow up. This findings are in line with evidence from other settings which highlighted stigma as a barrier to ART adherence (Nawa et al., 2008, Biadgilign et al., 2009, Bezabhe et al., 2014 and Wasti et al., 2012). 
Beliefs that alternative medicines (traditional herbs) can cure HIV was another obstacle to good ART adherence found by our study. Clients who use traditional herbs are likely to cause them to abandon their ART, poor cooperation with health care providers at the facility and consequently poor involvement in care. Other studies also highlight the use of alternative medicines as a major barrier to ART adherence (Nyogea et al., 2015, Biadgilign et al., 2009 and Kagee, 2012).

Our study limitations were; Intra-individual bias since some of the participants were used for both qualitative and quantitative data. However, we were confident about the findings of the study because we were able to reach saturation as most of the themes appeared repetitively.

\section{Conclusion}

Though CCLADs is a recommendable intervention that has improved ART accessibility among PLWHIV in their communities, more interventions aiming at ensuring full pill uptake among patients in CCLADs at LRRH are still necessary.

\section{Abbreviations}

CCLAD Community Client-Led Art Delivery

HIV Human immunodeficiency virus

AIDs Acquired immunodeficiency syndrome

LRRH Lira regional referral hospital

LIDC Lira infectious disease clinic

ART Anti-retroviral treatment

\section{Declarations}

\section{Ethics approval and consent to participate}

The authors confirm that the approvals were sought from appropriate institutions and all methods in this study were performed in accordance with the relevant guidelines and regulations. The study protocol was reviewed and cleared by the Gulu University Research Ethics Committee (GUREC-048-20). The protocol was further cleared for collection of data in Uganda by National research regulator, Uganda National Council of science and Technology (RESCLEAR/01). And administrative clearance for this study was obtained from the district health officer of Lira district and hospital administration of LRRH. Informed consent was obtained from all study participants. None of the participants was below 18 years of age. 


\section{Consent for publication}

Not applicable

\section{Availability of data and materials}

We can confirm that he datasets used and/or analysed during the current study will be available from the corresponding author on reasonable request.

\section{Competing interests}

The authors declare that they have no conflict of interest.

\section{Funding source}

"Research reported in this publication was supported by the Fogarty International Center (U.S. Department of State's Office of the U.S. Global AIDS Coordinator and Health Diplomacy [S/GAC] and the President's Emergency Plan for AIDS Relief [PEPFAR]) of the National Institutes of Health under Award Number R25TW011210. The content is solely the responsibility of the authors and does not necessarily represent the official views of the National Institutes of Health"

\section{Author contribution}

RI: Concept development, proposal development, data collection, data analysis, manuscript writing.

PSS: Proposal development, data collection, data analysis, manuscript writing.

DJO: Concept development, proposal development, data collection, data analysis, manuscript writing.

SOE: Proposal development, data collection

AK: Proposal development

LL: Concept development, proposal development

RI: Concept development

JS: Proposal development, manuscript writing

EK: Proposal development, manuscript writing

CO: Proposal development and manuscript writing 
All authors read and approved the manuscript for publication.

\section{Acknowledgments}

We give great thanks to the HEPI-TUITAH project administration for the spectacular job in translating our research vision into reality

In addition, we would like to express our gratitude to staff at ART clinic of LRRH more especially Dr. Luka Joshua who guided us in the entire recruitment process of respondents.

Finally, we are deeply appreciative to Dr. Moses Ocan and Dr. Andrew K. Turiho for their expertise and guidance through manuscript writing.

\section{References}

1. a qualitative study. (2008). 5(3), 136-143.

2. Barker, C., Dutta, A., \& Klein, K. (2017). Can differentiated care models solve the crisis in HIV treatment financing? Analysis of prospects for 38 countries in sub-Saharan Africa. Journal of the International AIDS Society. https://doi.org/10.7448/IAS.20.5.21648

3. Bezabhe, W. M., Chalmers, L., Bereznicki, L. R., Peterson, G. M., Bimirew, M. A., \& Kassie, D. M. (2014). Barriers and facilitators of adherence to antiretroviral drug therapy and retention in care among adult HIV-positive patients: A qualitative study from Ethiopia. PLOS ONE, 9(5). https://doi.org/10.1371/journal.pone.0097353

4. Biadgilign, S., Deribew, A., Amberbir, A., \& Deribe, K. (2009). Barriers and facilitators to antiretroviral medication adherence among HIV-infected paediatric patients in Ethiopia: A qualitative study. Sahara J, 6(4), 148-154. https://doi.org/10.1080/17290376.2009.9724943

5. Geng, E. H., \& Holmes, C. B. (2019). Research to improve differentiated hiv service delivery interventions: Learning to learn as we do. In PLoS Medicine. https://doi.org/10.1371/journal.pmed.1002809

6. Grimsrud, A., Bygrave, H., Doherty, M., Ehrenkranz, P., Ellman, T., Ferris, R., Ford, N., Killingo, B., Mabote, L., Mansell, T., Reinisch, A., Zulu, I., \& Bekker, L. G. (2016). Reimagining HIV service delivery: The role of differentiated care from prevention to suppression: The. In Journal of the International AIDS Society. https://doi.org/10.7448/IAS.19.1.21484

7. Kagimu D, Egessa J, Oucul L, S. E. (2018). Overcoming barriers to access of HIV/AIDS services among female sex workers through differentiated service delivery models, TAS O Entebbe experience. July, 2018.

8. Manuscript, A. (2012). NIH Public Access. 6(1), 1-12.

https://doi.org/10.1080/17441691003796387.Structural 
9. Mills, E. J., Nachega, J. B., Bangsberg, D. R., Singh, S., Rachlis, B., Wu, P., Wilson, K., Buchan, I., Gill, C. J., \& Cooper, C. (2006). Adherence to HAART: A Systematic Review of Developed and Developing Nation Patient-Reported Barriers and Facilitators. 3(11).

https://doi.org/10.1371/journal.pmed.0030438

10. Nyogea, D., Mtenga, S., Henning, L., Franzeck, F. C., Glass, T. R., \& Letang, E. (2015). Determinants of antiretroviral adherence among HIV positive children and teenagers in rural Tanzania: a mixed methods study. February. https://doi.org/10.1186/s12879-015-0753-y

11. Odongo, C. O., Bisaso, R. K., Byamugisha, J., \& Obua, C. (2014). Intermittent use of sulphadoxinepyrimethamine for malaria prevention: A cross-sectional study of knowledge and practices among Ugandan women attending an urban antenatal clinic. Malaria Journal. https://doi.org/10.1186/1475-2875-13-399

12. Ridgeway, K., Dulli, L. S., Murray, K. R., Silverstein, H., Santo, L. D., Olsen, P., De Mora, D. D., \& McCarraher, D. R. (2018). Interventions to improve antiretroviral therapy adherence among adolescents in low- and middle-income countries: A systematic review of the literature. In PLOS ONE (Vol. 13, Issue 1). https://doi.org/10.1371/journal.pone.0189770

13. Roy, M., Bolton Moore, C., Sikazwe, I., \& Holmes, C. B. (2019). A Review of Differentiated Service Delivery for HIV Treatment: Effectiveness, Mechanisms, Targeting, and Scale. In Current HIV/AIDS Reports. https://doi.org/10.1007/s11904-019-00454-5

14. (2015). Understanding Fast-Track Targets. Accelerating action to end the AIDS epidemic by 2030. Unaids, 12.

15. (2019). 2018 Global Hiv Statistics. Unaids, 1-6.

16. Wasti, S. P., Simkhada, P., Randall, J., Freeman, J. V., \& van Teijlingen, E. (2012). Factors influencing adherence to antiretroviral treatment in Nepal: A mixed-methods study. PLoS ONE, 7(5), 1-11. https://doi.org/10.1371/journal.pone.0035547

17. Weiser, S. D., Palar, K., Frongillo, E. A., Tsai, A. C., Hunt, P. W., Ragland, K., \& Martin, J. (2014). HHS Public Access Author manuscript AIDS. Author manuscript; available in PMC 2015 November 02. Published in final edited form as: AIDS. 2014 January 2; 28(1): 115-120. doi:10.1097/01.aids.0000433238.93986.35. Longitudinal assessment of associations betwe. Aids, 28(1), 115-120. https://doi.org/10.1097/01.aids.0000433238.93986.35.Longitudinal

18. Zakumumpa, H., Rujumba, J., Kwiringira, J., Katureebe, C., \& Spicer, N. (2020). Understanding implementation barriers in the national scale-up of differentiated ART delivery in Uganda. 1-16.

\section{Figures}


Pill adherence following a four day pill uptake recall

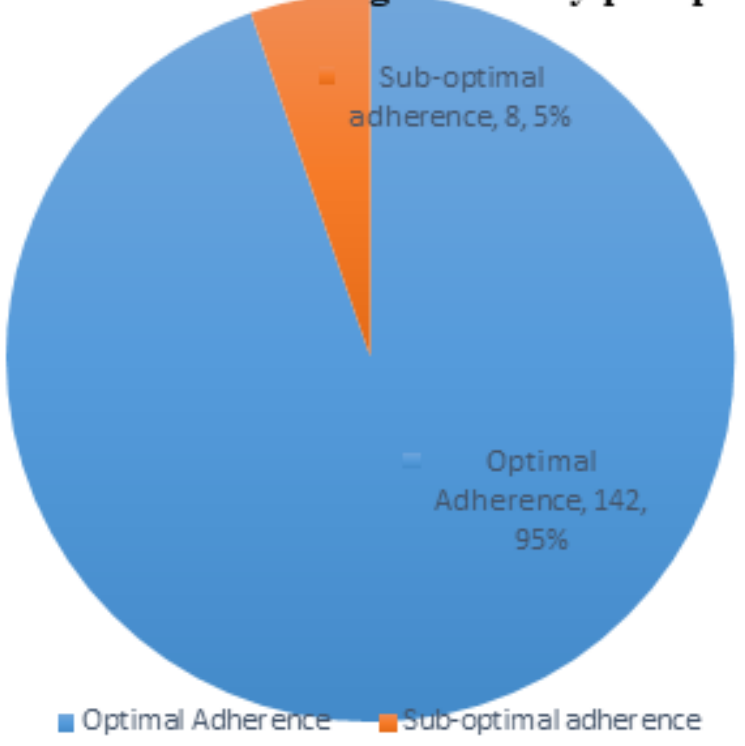

\section{Figure 1}

Pill adherence following a four day pill uptake retake 